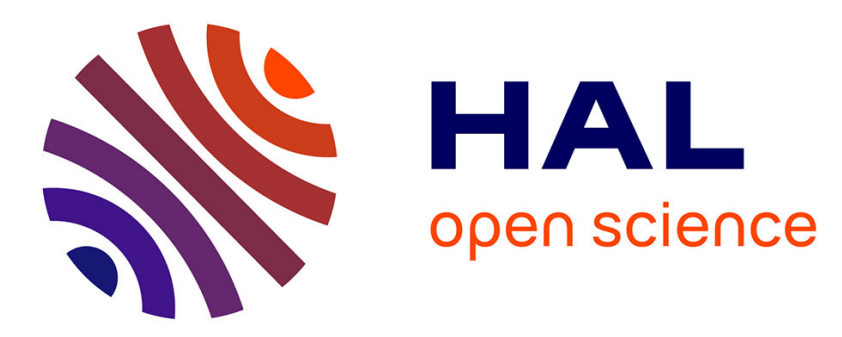

\title{
A homogenization-based damage model for stiffness loss in ductile metal-matrix composites
}

\author{
Khaoula Dorhmi, Leo Morin, Katell Derrien, Zehoua Hadjem-Hamouche, \\ Jean-Pierre Chevalier
}

\section{To cite this version:}

Khaoula Dorhmi, Leo Morin, Katell Derrien, Zehoua Hadjem-Hamouche, Jean-Pierre Chevalier. A homogenization-based damage model for stiffness loss in ductile metal-matrix composites. Journal of the Mechanics and Physics of Solids, 2020, 137, pp.1-16. 10.1016/j.jmps.2019.103812 . hal-02532883

\section{HAL Id: hal-02532883 \\ https://hal.science/hal-02532883}

Submitted on 6 Apr 2020

HAL is a multi-disciplinary open access archive for the deposit and dissemination of scientific research documents, whether they are published or not. The documents may come from teaching and research institutions in France or abroad, or from public or private research centers.
L'archive ouverte pluridisciplinaire HAL, est destinée au dépôt et à la diffusion de documents scientifiques de niveau recherche, publiés ou non, émanant des établissements d'enseignement et de recherche français ou étrangers, des laboratoires publics ou privés. 


\title{
A homogenization-based damage model for stiffness loss in ductile metal-matrix composites
}

\author{
Khaoula Dorhmi, Léo Morin*, Katell Derrien, Zehoua Hadjem-Hamouche, \\ Jean-Pierre Chevalier
}

PIMM, Arts et Metiers Institute of Technology, CNRS, Cnam, HESAM University, 151 boulevard de l'Hopital, Paris 75013, France

Keywords:

Metal-matrix composites

Ductile damage

Loss of stiffness

Homogenization

\begin{abstract}
A B S T R A C T
The aim of this paper is to develop a homogenized model that permits to describe the progressive loss of stiffness observed experimentally on ductile metal-matrix composites subjected to mechanical loadings. A two-step homogenization procedure is proposed to describe the effect of damage on the elastic properties. Since damage is solely driven by plastic deformation, a Gurson-type model is considered to describe the nucleation and growth of voids. Completed by appropriate evolution equations of the microstructure, this model of plasticity is used to update the elastic properties that are described by a meanfield homogenization scheme. The complete incremental model is implemented numerically and applied to the prediction of the stiffness loss. The model predictions are in very good agreement with experimental results on Al-SiC composites, cast irons and steel composites.
\end{abstract}

\section{Introduction}

Metal matrix composites are attractive materials whose industrial applications keep growing (Ibrahim et al., 1991; Mortensen and LLorca, 2010). This class of materials, which encompasses a large number of possibilities due to the base metal (e.g., aluminum, steel, copper), the shape (e.g. fibers, particles, whiskers) and type (e.g. carbides, nitrides) of reinforcements, as well as the manufacturing process (e.g. eutectic solidification, powder metallurgy, stir casting), can lead to significant enhancements in terms of mechanical and physical properties including high tensile and fatigue properties.

In structural applications, a classical property that is aimed at is the specific modulus, which is defined as the ratio between the elastic modulus $E$ and the density $\rho(E / \rho)$. Materials with high specific modulus are notably natural candidates in lightweight structures and are thus of important interest in automotive and aeronautic industries to improve energy efficiency and decrease $\mathrm{CO}_{2}$ emissions. In this context, novel metal matrix composites have emerged in the last decade; this has led for instance to the development of Fe-TiB 2 composites (Gaspérini et al., 2017; Hadjem-Hamouche et al., 2012, 2018) which are interesting for industrial purposes due to the very high elastic modulus of light $\mathrm{TiB}_{2}$ particles $\left(\mathrm{Olk}_{\mathrm{B}} \mathrm{moto}\right.$ et al., 2010); a volume fraction of $13 \% \mathrm{TiB}_{2}$ particles leads to a significant increase of the specific stiffness by about $15 \%$ (Hadjem-Hamouche et al., 2018).

The main limitation of metal matrix composites in structural applications is the embrittlement due to the presence of brittle ceramic reinforcements (Mortensen and LLorca, 2010). The presence of particles with high volume fraction promotes

\footnotetext{
* Corresponding author.

E-mail address: leo.morin@ensam.eu (L. Morin).
} 
the nucleation of damage in the form of particle cracking and interface decohesion on the metal matrix (Babout et al., 2001, 2004; Brechet et al., 1991; Hadjem-Hamouche et al., 2018; Maire et al., 2007) in addition to classical nucleation on second-phase particles. These mechanisms are directly associated with the presence of voids so that a notable consequence of the damage occurrence on the mechanical properties, prior to the final failure, is a decrease of the elastic modulus: in the case of an $\mathrm{Al}-\mathrm{SiC}$ composite (with $15 \%$ of SiC particles), a decrease of about $10 \%$ of the elastic modulus was observed (Derrien et al., 1999) after about 5\% of deformation. Thus, structural metal-matrix composites are mainly limited in real applications because they may lose their improved properties in processes based on plastic deformation such as metal forming and rolling. The main challenge posed by metal matrix composites is to find how the microstructure of these materials evolves during plastic straining in order to design optimal processing paths that permit to minimize the damage occurrence.

In terms of modeling, the mechanical response of metal-matrix composites has been mainly studied by computational micromechanics. Numerical finite element cell models (Christman et al., 1989; Legarth and Niordson, 2010; LLorca and González, 1998; LLorca et al., 1991; Niordson and Tvergaard, 2001; Tvergaard, 1990), consisting in a single particle embedded in a matrix subjected to periodic boundary conditions, have shown the effect of particle morphology and volume fraction on the overall mechanical response as well as the importance of stress triaxiality. The effect of microstructure and particle clustering have been also investigated on elastic (Michel et al., 1999; Mishnaevsky et al., 2004; Moulinec and Suquet, 1998; Segurado and LLorca, 2002) and plastic properties (Chawla and Chawla, 2006; Ghosh and Moorthy, 1998; Segurado et al., 2003; Shakoor et al., 2018). The analytical modeling of metal-matrix composites have mainly focused on the derivation of elastic properties using mean-field approaches (Segurado and LLorca, 2002) and plastic properties without damage (Doghri and Ouaar, 2003; González and LLorca, 2000; Mueller and Mortensen, 2006; Ponte Castañeda and Suquet, 1997). Owing to the complexity of the local mechanisms responsible for deformation and fracture (e.g. particle cracking, matrix/particles interface decohesion), the derivation of models that include damage is scarce. Phenomenological (Voyiadjis and Kattan, 1993) and micromechanical (Derrien et al., 1999; González and LLorca, 2000; LLorca et al., 1991; Tekoglu and Pardoen, 2010) models have permitted to reproduce accurately macroscopic stress-strain curves but they do not permit to predict the loss of stiffness observed after plastic straining. This drawback is due to an inaccurate description of the coupling between elasticity, plasticity and damage that requires a multiscale model able to account explicitly for microstructural changes.

The aim of this paper is to derive a micromechanical model for ductile metal-matrix composites that is able to describe the progressive loss of stiffness observed during plastic straining, based on microstructure changes. The problem of stiffness loss, which has been always disregarded in ductile failure modeling, requires the development of a micromechanical model that needs to contain internal parameters describing the elastic behavior in terms of the existing phases and allow the description of the possible evolution of the phases together with the initiation of damage. The paper is organized as follows. In Section 2, a two-step homogenization is performed which permits to derive a three-phases elastic model incorporating the influence of particles, matrix and voids, together with a plastic model which describes the nucleation and growth of voids responsible for the stiffness loss. An efficient algorithm for the local step of the elastoplastic solution is presented in Section 3 in order to provide a finite element implementation of the model. In Section 4 the predictions of the model are finally compared to experimental results on aluminum, cast iron and steel composites which show stiffness loss during plastic straining.

\section{A two-step homogenized model for ductile elastic-plastic metal-matrix composites}

\subsection{Generalities}

Our aim is to derive a model that is able to describe the progressive loss of stiffness of a metal-matrix composite due to damage accumulation during plastic deformation. In metals, damage is mainly due to the presence of voids, that can be nucleated on primary particles or in the matrix (second-phase particles), which will grow and ultimately induce macroscopic cracks. Thus we are looking for an elastic-plastic model that describes (i) the basic features of ductile failure (nucleation, growth and coalescence of voids) and (ii) the loss of stiffness due to damage. In order to account for these two phenomena, it is necessary to derive a micromechanical model accounting for the microstructure of the metal-matrix composite to provide overall elastic properties, and its possible evolution during plastic straining.

In metal-matrix composites, damage nucleation can arise from three sources (Babout et al., 2001, 2004; Brechet et al., 1991; Hadjem-Hamouche et al., 2018; Maire et al., 2007) (see Fig. 1): (i) the decohesion of the interface between reinforcements (primary particles) and the matrix, (ii) the cracking of reinforcements and (iii) the nucleation within the matrix on second-phase particles. In this work, damage is supposed to be driven only by plastic deformation so no microstructure modifications are induced by elastic strains. With this assumption, it is possible to treat separately the elastic and plastic behaviors in a two-step homogenization procedure:

- The elastic behavior is supposed not to induce damage so the overall elastic properties will only depend on the microstructure (distribution and volume fraction of various phases) at a given instant. 

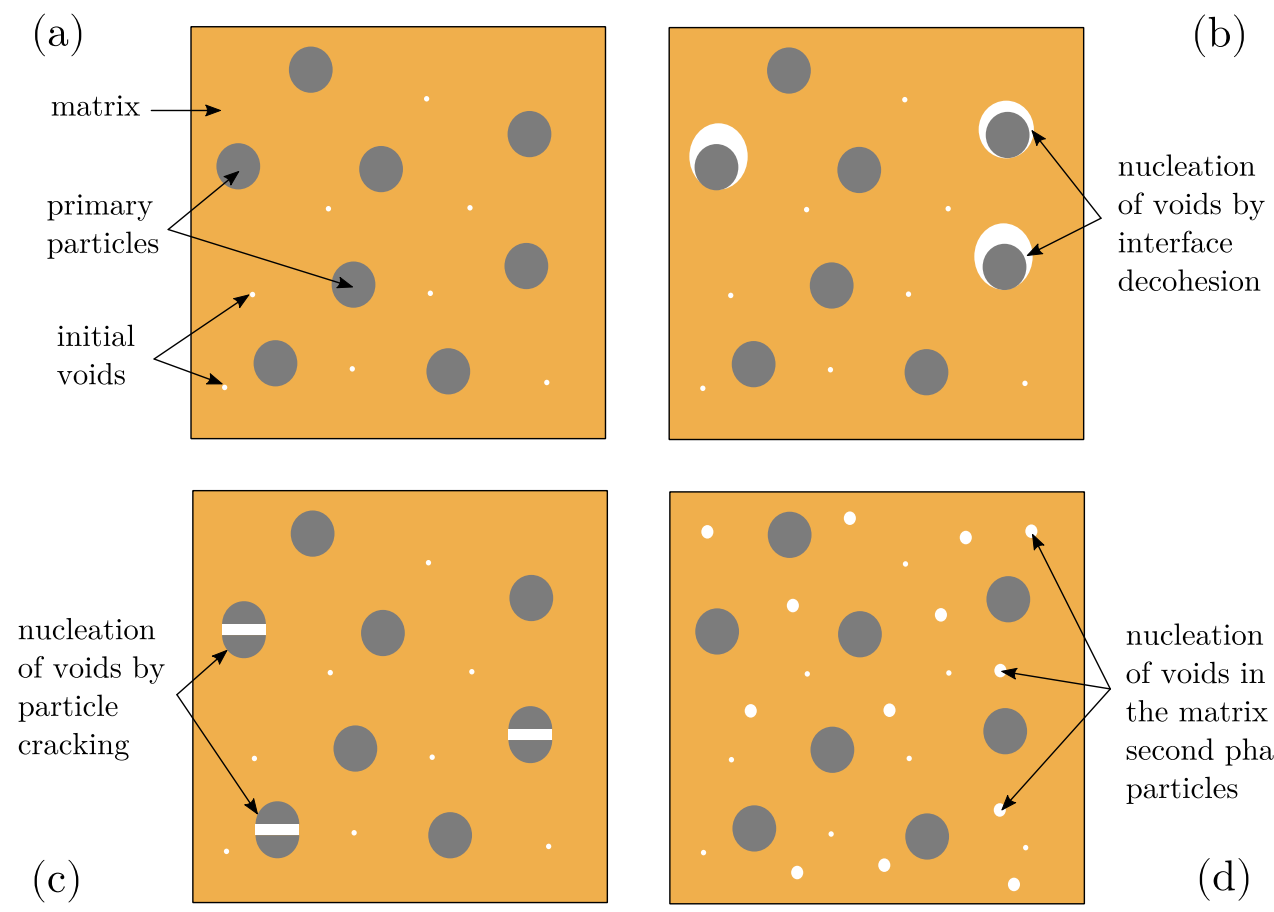

nucleation of voids in the matrix on second phase particles

(d)

Fig. 1. Damage mechanisms in metal-matrix composites: (a) Initial configuration with sound particles and initial voids, (b) Damaged configuration with nucleation of voids by interface decohesion, (c) Damaged configuration with nucleation of voids by particle cracking, (d) Damaged configuration with nucleation of voids in the matrix on second-phase particles.

- The plastic behavior is supposed to induce damage through the nucleation and growth of voids due to plastic straining. The consequence of the evolution of damage is a modification of the microstructure which would ultimately modify incrementally the elastic properties.

\subsection{Homogenization of elastic properties}

As just explained, the elastic behavior is supposed not to induce damage. Thus, at a given instant, the macroscopic elastic behavior only depends on the nature and distribution of the phases of the actual microstructure. In the very general case of a $N$-phase composites, made of a matrix characterized by its volume fraction $f^{m}$ and its stiffness tensor $\mathbb{C}_{m}$, and $N-1$ secondary phases (which may be particles or voids) characterized by their volume fraction $f^{r}$ and their stiffness tensor $\mathbb{C}_{r}$ (with $1 \leq r \leq N$ ), the overall stiffness tensor $\overline{\mathbb{C}}$ can be estimated accurately by mean-field approaches of homogenization. In the case of Mori and Tanaka (1973)'s scheme, which is relevant for low to moderate volume fractions of secondary phases, the overall stiffness tensor $\overline{\mathbb{C}}$ is given by

$$
\overline{\mathbb{C}}=\mathbb{C}_{m}+\sum_{r=1}^{N} f^{r}\left(\mathbb{C}_{r}-\mathbb{C}_{m}\right): \mathbb{A}_{r},
$$

where $\mathbb{A}_{r}$ is the localization tensor that reads

$$
\mathbb{A}_{r}=\left(\mathbb{I}+\mathbb{S}^{r}: \mathbb{C}_{m}^{-1}:\left(\mathbb{C}_{r}-\mathbb{C}_{m}\right)\right)^{-1} .
$$

In this equation, $\mathbb{I}$ is the fourth-order identity tensor and $\mathbb{S}^{r}$ is the (classical) Eshelby tensor associated to the $r$-th phase.

In most of metal-matrix composites, only one family of particles is involved which permits to simplify the number of phases. In the following we thus consider the simplified case of a 3-phase composite made of a matrix, particles and cavities, which allows to account for the presence of damage in the metal-matrix composite. For purposes of simplicity, additional assumptions are made: (i) all phases are supposed to be elastically isotropic and (ii) particles and voids are supposed to be spherical. The assumption of a spherical shape for the particles is reasonable in most metal-matrix composites with moderate shape effects. However, in the case of fiber-reinforced composites, which is not considered in this work, this assumption is not appropriate and it would be necessary to consider other types of geometry such as elongated ellipsoids or cylinders. The hypothesis of spherical void is also reasonable in most cases since spherical particles are likely to produce nucleated spherical voids. During plastic deformation, spherical voids are supposed to stay spherical, which is relevant in the case of moderate to high stress triaxiality. These assumptions will allow the derivation of closed-form expressions for the macroscopic elastic moduli. 
We are thus investigating the overall behavior of a 3-phase composite made of an isotropic matrix characterized by its shear and bulk moduli $\mu_{m}$ and $\kappa_{m}$, reinforced by isotropic spherical particles characterized by their shear and bulk moduli $\mu_{p}$ and $\kappa_{p}$, and containing spherical voids. The volume fractions of the matrix, particles and voids are respectively denoted by $f^{m}, f^{p}$ and $f^{v}$, where $f^{m}+f^{p}+f^{v}=1$. By using the previous assumptions, it can be shown that the overall stiffness tensor $\overline{\mathbb{C}}$ given by (1) and (2) reduces to

$$
\overline{\mathbb{C}}=3 \bar{\kappa} \mathbb{J}+2 \bar{\mu} \mathbb{K}
$$

where $\mathbb{J}$ is the spherical projection tensor and $\mathbb{K}$ the deviatoric projection tensor. The bulk modulus $\bar{\kappa}$ and shear modulus $\bar{\mu}$ are given by (Mori and Tanaka, 1973)

$$
\left\{\begin{array}{l}
\bar{\kappa}=\kappa_{m} \times \frac{f^{m}\left(\kappa_{m}+\alpha_{m}\left(\kappa_{p}-\kappa_{m}\right)\right)+f^{p} \kappa_{p}}{f^{m}\left(\kappa_{m}+\alpha_{m}\left(\kappa_{p}-\kappa_{m}\right)\right)+f^{p} \kappa_{m}+\frac{f^{v}}{1-\alpha_{m}}\left(\kappa_{m}+\alpha_{m}\left(\kappa_{p}-\kappa_{m}\right)\right)} \\
\bar{\mu}=\mu_{m} \times \frac{f^{m}\left(\mu_{m}+\beta_{m}\left(\mu_{p}-\mu_{m}\right)\right)+f^{p} \mu_{p}}{f^{m}\left(\mu_{m}+\beta_{m}\left(\mu_{p}-\mu_{m}\right)\right)+f^{p} \mu_{m}+\frac{f^{v}}{1-\beta_{m}}\left(\mu_{m}+\beta_{m}\left(\mu_{p}-\mu_{m}\right)\right)},
\end{array}\right.
$$

where $\alpha_{m}$ and $\beta_{m}$ are given by

$$
\left\{\begin{array}{l}
\alpha_{m}=\frac{3 \kappa_{m}}{3 \kappa_{m}+4 \mu_{m}} \\
\beta_{m}=\frac{6}{5} \frac{\kappa_{m}+2 \mu_{m}}{3 \kappa_{m}+4 \mu_{m}} .
\end{array}\right.
$$

The macroscopic Young's modulus and Poisson ratio which are denoted by $\bar{E}$ and $\bar{v}$ are finally given by

$$
\left\{\begin{array}{l}
\bar{E}=\frac{9 \bar{\kappa} \bar{\mu}}{3 \bar{\kappa}+\bar{\mu}} \\
\bar{v}=\frac{3 \bar{\kappa}-2 \bar{\mu}}{2(3 \bar{\kappa}+\bar{\mu})} .
\end{array}\right.
$$

The elastic properties thus depend only on (i) the elastic properties of the phases through $\kappa_{m}, \mu_{m}, \kappa_{p}$ and $\mu_{p}$ and (ii) the volume fraction of the phases through $f^{m}, f^{p}$ and $f^{v}$. A modification of the microstructure (due to plastic straining for instance), which corresponds in practice to a modification of the volume fraction of the phases, will consequently modify the macroscopic properties.

\subsection{Homogenization of plastic properties}

The plastic behavior of the metal-matrix composite is an important step in the construction of the complete model since it will drive the evolution of the microstructure due to damage initiation and accumulation. The plasticity model should (i) describe the basic features of ductile failure (nucleation, growth and coalescence of microvoids) and (ii) provide evolution laws for the internal parameters defining the microstructure (volume fractions of matrix, reinforcements and voids).

Plastic behavior without voids. We assume that (i) the matrix follows an isotropic von Mises yield criterion with a yield stress denoted by $\sigma_{0}^{m}$ and (ii) the particles remain elastic. The consequence of elastic particles is a modification of the local stress field in the matrix, which can ultimately modify the onset of plasticity. Thus, it is reasonable to assume that, since particles are isotropic, the system \{matrix+particles\} also follows an isotropic von Mises yield criterion of the form

$$
\varphi(\sigma)=\sigma_{e q}-\sigma_{0} \leq 0
$$

where $\sigma_{e q}$ is the overall equivalent stress in the system \{matrix+particles and $\sigma_{0}$ is identified as the yield stress of the sound composite. No attempt is made here to connect the yield stress of the composite $\sigma_{0}$ with the yield stress of the sole matrix $\sigma_{0}^{m}$. The reason for this choice is that, in the derivation of the plastic behavior with voids, only the plastic behavior of the sound composite will be needed. The determination of the yield stress $\sigma_{0}$ can then be easily done from experimental tensile tests on the composite.

Plastic behavior with voids. We now consider the presence of voids, by following the approach of Gurson (1977), based on the limit-analysis of a hollow sphere made of an isotropic von Mises plastic matrix (see Fig. 2). This model is suitable to describe the plastic behavior of a porous material and the increase of porosity due to plastic flow of the plastic matrix. It is important to note that the term "matrix" considered in Gurson's analysis does not have the same meaning than the matrix of the metal-matrix composite: it just corresponds, in Gurson's paper, to some plastic material (obeying von Mises criterion) surrounding the spherical void. Here, Gurson's matrix can be identified as the local plastic potential (7) describing the plasticity of the sound metal-matrix composite, and will be called "homogenized matrix" in the following to avoid any ambiguity. The volume fraction of voids is not changed and remains denoted by $f^{v}$.

The macroscopic yield criterion, expressed in term of the macroscopic stress $\Sigma$, reads (Gurson, 1977)

$$
\Phi(\Sigma)=\frac{\Sigma_{e q}^{2}}{\sigma_{0}^{2}}+2 q_{1} f^{v} \cosh \left(\frac{3 q_{2} \Sigma_{m}}{2 \sigma_{0}}\right)-1-q_{3}\left(f^{v}\right)^{2} \leq 0,
$$




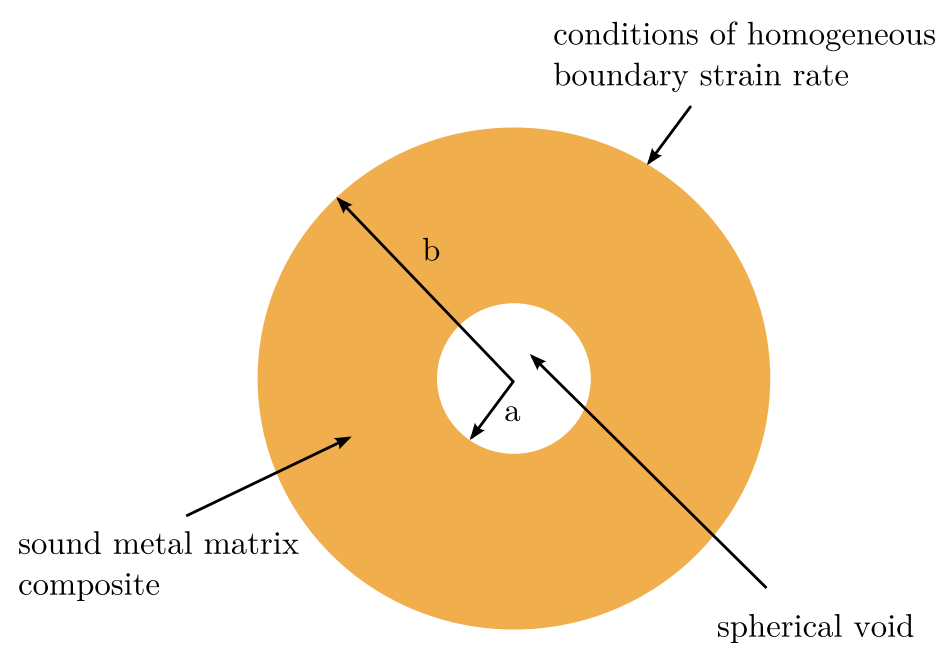

Fig. 2. Elementary cell considered by Gurson (1977) for the plastic behavior.

where $\Sigma_{e q}$ is the macroscopic equivalent von Mises stress, $\Sigma_{m}$ is the macroscopic mean stress and $\sigma_{0}$ is the yield stress of the homogenized matrix. The parameters $q_{1}, q_{2}$ and $q_{3}$ are (classical) Tvergaard's parameters (Tvergaard, 1981; Tvergaard and Needleman, 1984) that permit to improve the agreement between the predictions of Gurson (1977)'s model and the results of micromechanical simulations of ductile failure on elementary cells with more realistic shapes than Gurson (1977)'s hollow sphere.

The macroscopic yield criterion is completed by a macroscopic flow rule obtained from the property of normality which is preserved during scale transition:

$$
\mathbf{D}^{\mathrm{p}}=\dot{\lambda} \frac{\partial \Phi}{\partial \boldsymbol{\Sigma}}(\boldsymbol{\Sigma}), \quad \dot{\lambda}\left\{\begin{array}{lll}
=0 & \text { if } & \Phi(\boldsymbol{\Sigma})<0 \\
\geq 0 & \text { if } & \Phi(\boldsymbol{\Sigma})=0
\end{array}\right.
$$

where $\mathbf{D}^{\mathrm{p}}$ denotes the plastic Eulerian strain rate and $\dot{\lambda}$ the plastic multiplier.

Hardening is accounted for using Gurson (1977)'s heuristic approach; the constant yield stress $\sigma_{0}$ in the criterion (8) is replaced by some average yield stress $\bar{\sigma}$ given by

$$
\bar{\sigma}=\sigma(\bar{\epsilon}),
$$

where $\sigma(\epsilon)$ is the hardening function providing the yield limit as a function of the cumulated plastic strain $\epsilon$, and $\bar{\epsilon}$ represents some average equivalent strain in the homogenized matrix. The evolution of $\bar{\epsilon}$ is given by the following equation

$$
\left(1-f^{v}\right) \bar{\sigma} \dot{\bar{\epsilon}}=\boldsymbol{\Sigma}: \mathbf{D}^{p},
$$

which corresponds to the (heuristic) assumption proposed by Gurson (1977) that the plastic dissipation in the porous material, $\boldsymbol{\Sigma}: \mathbf{D}^{p}$, is equal to that of a fictitious homogeneous material.

Finally, the evolution equation of the porosity corresponding to void growth is classically deduced from the incompressibility of the matrix:

$$
\dot{f}_{\text {growth }}=\left(1-f^{v}\right) \operatorname{tr} \mathbf{D}^{p} .
$$

Void nucleation. Void nucleation is assumed to be described by the phenomenological model of Chu and Needleman (1980) and Needleman (1987). This model, derived from micromechanical cell analyses, provides in its general form the rate of void nucleation as a function of the strain and stress rates; it is thus composed of strain-controlled and stresscontrolled nucleation terms. In practice, the stress-controlled term is generally disregarded because it is sensitive to instabilities due to its dependance on the hydrostatic stress which favors early flow localization (Benzerga and Leblond, 2010; Benzerga et al., 2016; Pan et al., 1983). On the other hand, strain-controlled term, although not associated with an energy criterion, is generally considered to be sufficient to capture macroscopically void nucleation (Benzerga and Leblond, 2010; Benzerga et al., 2016). Thus, in the following, we make the (classical) assumption that the nucleation rate is solely composed of a strain-controlled contribution.

As shown in Fig. 1, void nucleation, in the case of metal-matrix composites, can be the consequence of (i) the decohesion of matrix/particles interfaces, (ii) particle cracking and (iii) second-phase particles. Thus the total void nucleation rate $\dot{f}_{\text {nucleation }}$ is supposed to be of the form

$$
\dot{f}_{\text {nucleation }}=\dot{f}_{\text {decohesion }}+\dot{f}_{\text {cracking }}+\dot{f}_{\text {second-phase }} \text {. }
$$


In Eq. (13) each nucleation mechanism is supposed to be described independently by the strain-controlled contribution of Chu and Needleman (1980)'s model. The three contributions of the total nucleation rate $\dot{f}_{\text {decohesion }}, \dot{f}_{\text {cracking }}$ and $\dot{f}_{\text {second-phase }}$ follow a normal distribution and can be written as

$$
\left\{\begin{array}{l}
\dot{f}_{\text {decohesion }}=\frac{f_{N}^{\mathrm{d}}}{s_{N}^{\mathrm{d}} \sqrt{2 \pi}} \exp \left(-\frac{1}{2}\left(\frac{\bar{\epsilon}-\epsilon_{N}^{\mathrm{d}}}{s_{N}^{\mathrm{d}}}\right)^{2}\right) \times \dot{\bar{\epsilon}} \\
\dot{f}_{\text {cracking }}=\frac{f_{N}^{\mathrm{c}}}{s_{N}^{\mathrm{c}} \sqrt{2 \pi}} \exp \left(-\frac{1}{2}\left(\frac{\bar{\epsilon}-\epsilon_{N}^{\mathrm{c}}}{s_{N}^{\mathrm{c}}}\right)^{2}\right) \times \dot{\bar{\epsilon}} \\
\dot{f}_{\text {second-phase }}=\frac{f_{N}^{\mathrm{sp}}}{s_{N}^{\mathrm{sp}} \sqrt{2 \pi}} \exp \left(-\frac{1}{2}\left(\frac{\bar{\epsilon}-\epsilon_{N}^{\mathrm{sp}}}{s_{N}^{s p}}\right)^{2}\right) \times \dot{\bar{\epsilon}}
\end{array}\right.
$$

where $\bar{\epsilon}$ is the average plastic strain defined by Eq. (11), $f_{N}^{\mathrm{d}}, f_{N}^{\mathrm{c}}$ and $f_{N}^{\mathrm{sp}}$ represent the volume fractions of nucleating voids, $\epsilon_{N}^{\mathrm{d}}, \epsilon_{N}^{\mathrm{c}}$ and $\epsilon_{N}^{\mathrm{sp}}$ correspond to some average nucleation strains and $s_{N}^{\mathrm{d}}, s_{N}^{\mathrm{c}}$ and $s_{N}^{\mathrm{sp}}$ are standard deviations, respectively for the nucleation due to interface decohesion, particles cracking and second-phase particles.

We make the assumptions that (i) the average nucleation strains $\epsilon_{N}^{\mathrm{d}}, \epsilon_{N}^{\mathrm{c}}$ and $\epsilon_{N}^{\mathrm{sp}}$ are all equal to a single value $\epsilon_{N}$ and (ii) the standard deviations $s_{N}^{\mathrm{d}}, s_{N}^{\mathrm{c}}$ and $s_{N}^{\mathrm{sp}}$ are all equal to a single value $s_{N}$. These assumptions are quite reasonable in real applications for several reasons. First, it seems difficult, if not impossible, to provide an experimental identification of the contribution of each nucleation source upon the nucleation strain and the standard deviation; a single value for these parameters appears to be reasonable. This assumption reduces the number of nucleation parameters by focusing on the most relevant material parameters, which are the volume fractions of nucleating voids. This decrease in the number of parameters will be notably profitable to facilitate the calibration and the numerical integration of the model. Finally, this permits, for the nucleation model, to be compatible with the void growth model which concerns only one population of cavities. Indeed, several nucleation strains and standard deviations would imply that different populations of cavities would nucleate, which is incompatible with the void growth model considered.

Under these assumptions, the partial nucleation rates can thus be written in terms of a total nucleation rate $\dot{f}_{\text {nucleation }}$ such as

$$
\dot{f}_{\text {decohesion }}=\theta^{\mathrm{d}} \dot{f}_{\text {nucleation }}, \quad \dot{f}_{\text {cracking }}=\theta^{\mathrm{c}} \dot{f}_{\text {nucleation }}, \quad \dot{f}_{\text {second-phase }}=\theta^{\text {sp }} \dot{f}_{\text {nucleation }},
$$

where the parameters $\theta^{\mathrm{d}}, \theta^{\mathrm{c}}$ and $\theta^{\mathrm{sp}}$ correspond to the fractional contributions of each nucleation process to the overall volume fraction of nucleating particles denoted by $f_{N}$ :

$$
\theta^{\mathrm{d}}=\frac{f_{N}^{\mathrm{d}}}{f_{N}}, \quad \theta^{\mathrm{c}}=\frac{f_{N}^{\mathrm{c}}}{f_{N}}, \quad \theta^{\mathrm{sp}}=\frac{f_{N}^{\mathrm{sp}}}{f_{N}}, \quad \theta^{\mathrm{d}}+\theta^{\mathrm{c}}+\theta^{\mathrm{sp}}=1 .
$$

In Eq. (15) the total nucleation rate is simply given by

$$
\dot{f}_{\text {nucleation }}=\frac{f_{N}}{s_{N} \sqrt{2 \pi}} \exp \left[-\frac{1}{2}\left(\frac{\bar{\epsilon}-\epsilon_{N}}{s_{N}}\right)^{2}\right] \times \dot{\bar{\epsilon}}
$$

where $f_{N}, \epsilon_{N}$ and $s_{N}$ respectively represent the volume fraction, average nucleation strain and standard deviation of the overall nucleating voids.

\subsection{Evolution of the microstructure}

The last step is now to connect the void growth rate $\dot{f}_{\text {growth }}$ and the partial nucleation rates $\dot{f}_{\text {decohesion }}, \dot{f}_{\text {cracking }}$ and $\dot{f}_{\text {second-phase }}$ to the volume fraction rates of the phases, $\dot{f}^{m}, \dot{f}^{p}$ and $\dot{f}^{v}$, which contribute to the overall elastic behavior.

General case. We make the assumptions that (i) the partial nucleation rate due to decohesion solely decreases the volume fraction of particles, (ii) the partial nucleation rate due to second-phase particles solely decreases the volume fraction of matrix and (iii) the partial nucleation rate due particle cracking may decrease (with a ratio to be set) both particles and matrix volume fractions.

Thus we assume that the evolution laws of the microstructure parameters are of the form

$$
\left\{\begin{array}{l}
\dot{f}^{v}=\dot{f}_{\text {growth }}+\dot{f}_{\text {nucleation }} \\
\dot{f}^{p}=-\dot{f}_{\text {decohesion }}-\gamma \dot{f}_{\text {cracking }} \\
\dot{f}^{m}=-\dot{f}_{\text {growth }}-\dot{f}_{\text {second-phase }}-(1-\gamma) \dot{f}_{\text {cracking }},
\end{array}\right.
$$

where the parameter $\gamma$ (with $0 \leq \gamma \leq 1$ ) permits to account for the fractional contribution of cracking to the decrease of particles and matrix volume fractions. The case $\gamma=0$ corresponds to a mechanism of particle cracking that only affects and decreases the volume fraction of particles while the case $\gamma=1$ corresponds to a mechanism that affects and decreases 
solely the volume fraction of the matrix. Intermediate values of $\gamma$, which combine both mechanisms, are more realistic in practice, because the effect of cracking is expected to affect both particles and matrix.

It should be noted that this modeling only permits to account approximately for the effect of cracking on the effective elastic properties; in practice, cracking would lead to an anisotropic elastic behavior depending on the crack morphology.

By taking advantage of Eqs. (15) and (17), the evolution laws of the microstructure parameters can be finally written as

$$
\left\{\begin{array}{l}
\dot{f}^{v}=\dot{f}_{\text {growth }}+\dot{f}_{\text {nucleation }} \\
\dot{f}^{p}=-\left(\theta^{\mathrm{d}}+\gamma \theta^{\mathrm{c}}\right) \dot{f}_{\text {nucleation }} \\
\dot{f}^{m}=-\dot{f}_{\text {growth }}-\left(\theta_{\mathrm{sp}}-(1-\gamma) \theta_{\mathrm{c}}\right) \dot{f}_{\text {nucleation }}=-\dot{f}_{\text {growth }}-\left(1-\left(\theta^{\mathrm{d}}+\gamma \theta^{\mathrm{c}}\right)\right) \dot{f}_{\text {nucleation }} \\
\theta^{\mathrm{d}}+\theta^{\mathrm{c}}+\theta^{\mathrm{sp}}=1, \quad 0 \leq \gamma \leq 1 .
\end{array}\right.
$$

A simplified model. We can notice that the consequences of nucleation on the microstructure rates depend only on a subtle combination of the fractional contributions of nucleation $\theta^{\mathrm{d}}, \theta^{\mathrm{c}}$ and $\theta^{\mathrm{sp}}$ and the parameter $\gamma$ through the ratio $\theta^{\mathrm{d}}+\gamma \theta^{\mathrm{c}}$. Thus if we assume that the values of the parameters $\theta^{\mathrm{d}}, \theta^{\mathrm{c}}$ and $\gamma$ are known, the ratio

$$
\theta=\theta^{\mathrm{d}}+\gamma \theta^{\mathrm{c}}
$$

is sufficient to characterize the evolution rates of the microstructure.

Thus, in practical situations where the calibration of $\theta^{\mathrm{d}}, \theta^{\mathrm{c}}$ and $\gamma$ is not possible, we propose a simplified model for the microstructure evolution based on the sole parameter $\theta$; the evolution equations of the microstructure read in that case

$$
\left\{\begin{array}{l}
\dot{f}^{v}=\dot{f}_{\text {growth }}+\dot{f}_{\text {nucleation }} \\
\dot{f}^{p}=-\theta \dot{f}_{\text {nucleation }} \\
\dot{f}^{m}=-\dot{f}_{\text {growth }}-(1-\theta) \dot{f}_{\text {nucleation }} .
\end{array}\right.
$$

The parameter $\theta$ combines several nucleation mechanisms and corresponds to the overall nucleation ratio that permits to distinguish nucleation within the matrix and the particles. This simplified model only looses the exact detail of the ratio of each nucleation source while keeping the main features of the full model. It reduces the number of internal parameters regarding nucleation to a strict minimum: three parameters defining the overall nucleation model $\left(f_{N}, \epsilon_{N}, s_{N}\right)$ and one parameter defining the ratio between nucleated voids within the matrix and the particles $(\theta)$. Some comments are in order regarding the limiting values of parameter $\theta$ :

- In the case of decohesion of matrix/particles interfaces $\left(\theta^{\mathrm{d}}=1\right)$, the overall nucleation ratio takes the value $\theta=1$;

- In the case of second-phase particles nucleation $\left(\theta^{\mathrm{sp}}=1\right)$, the overall nucleation ratio takes the value $\theta=0$.

In the case of void nucleation by particle cracking or a combination of several mechanisms, the parameter $\theta$ will take a value between 0 and 1 . The value of the parameter $\theta$ is expected to have a significant impact on stiffness loss when the volume fraction of particles is high and when the ratio between the matrix and particles moduli is important.

\section{Numerical implementation of the model}

The aim of this section is to provide a numerical implementation of the model. We focus on the local step of the elastoplastic solution which consists in projecting the elastic stress predictor on the yield surface.

\subsection{Generalities}

It has been shown in Enakoutsa et al. (2007) that Gurson (1977)'s model fits into the class of "generalized standard" materials (Halphen and Nguyen, 1975) under some hypotheses. This has important consequences regarding the numerical implementation of the model because, for this class of materials, the local projection problem has a unique solution provided that the internal parameters are discretized in time with an implicit scheme. In fact, in the case of Gurson-type models, the existence and uniqueness of the solution of the projection problem are ensured (Enakoutsa et al., 2007), provided that (i) the evolution equations of $\boldsymbol{\epsilon}$ (the total strain) and $\bar{\epsilon}$ (the hardening parameter) are discretized in time with an implicit-scheme; (ii) the additional terms due to the objective time-derivative of $\boldsymbol{\Sigma}$ (in the hypoelasticity law) are discretized in time with an explicit-scheme; (iii) the values of $f^{v}, f^{p}$ and $f^{m}$ used in the criterion, the flow rule and the elastic behavior are those at the preceding time-step. 


\subsection{Local projection problem}

For simplicity reasons, the algorithm is presented in the context of small strains; its extension to large strains does not raise any specific difficulties. The constitutive equations read

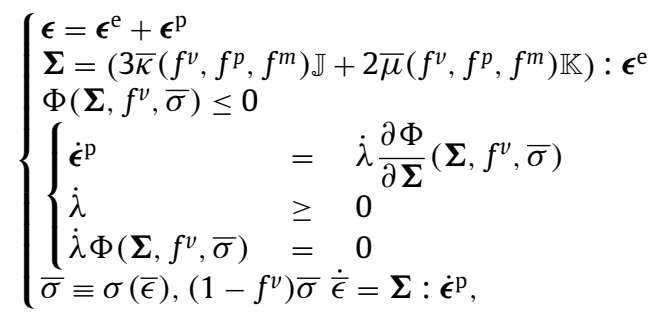
Decomposition of the total strain Isotropic elasticity law Plasticity criterion

Flow rule and consistency conditions

where $\boldsymbol{\epsilon}^{\mathrm{e}}$ is the elastic strain tensor, $\boldsymbol{\epsilon}^{\mathrm{P}}$ the plastic strain tensor.

The local projection problem thus consists in finding the mechanical state $\mathcal{S}_{n+1}=\left\{\boldsymbol{\epsilon}_{n+1}, \boldsymbol{\epsilon}_{n+1}^{\mathrm{p}}, \boldsymbol{\Sigma}_{n+1}, \bar{\epsilon}_{n+1}\right\}$ at time $t_{n+1}$ resulting from a given, known strain increment $\Delta \boldsymbol{\epsilon}_{n}$ (resulting from a global elastoplastic iteration), knowing the previous mechanical state $\mathcal{S}_{n}=\left\{\boldsymbol{\epsilon}_{n}, \boldsymbol{\epsilon}_{n}^{\mathrm{p}}, \boldsymbol{\Sigma}_{n}, \bar{\epsilon}_{n}\right\}$ at time $t_{n}$.

The yield criterion and the flow rule are thus discretized using an implicit scheme with respect to $\epsilon^{\mathrm{p}}$ and $\bar{\epsilon}$, while an explicit scheme is considered for $f^{v}, f^{p}$ and $f^{m}$. The discretized equations of the local projection problem are given by

$$
\begin{cases}\boldsymbol{\Sigma}_{n+1} & =\boldsymbol{\Sigma}_{n+1}^{\text {elas }}-\left(3 \bar{\kappa}_{n \mathbb{J}} \mathbb{J}+2 \bar{\mu}_{n} \mathbb{K}\right): \Delta \boldsymbol{\epsilon}_{n}^{\mathrm{p}} \\ \Phi\left(\boldsymbol{\Sigma}_{n+1}, f_{n}^{v}, \bar{\sigma}_{n+1}\right) & \leq 0 \\ \Delta \boldsymbol{\epsilon}_{n}^{\mathrm{p}} & =\Delta \lambda_{n} \frac{\partial \Phi}{\partial \boldsymbol{\Sigma}}\left(\boldsymbol{\Sigma}_{n+1}, f_{n}^{v}, \bar{\sigma}_{n+1}\right) \\ \Delta \lambda_{n} & \geq 0 \\ \Delta \lambda_{n} \Phi\left(\boldsymbol{\Sigma}_{n+1}, f_{n}, \bar{\sigma}_{n+1}\right) & =0 \\ \bar{\sigma}_{n+1} & =\sigma\left(\bar{\epsilon}_{n}+\Delta \bar{\epsilon}_{n}\right) \\ \left(1-f_{n}^{v}\right) \bar{\sigma}_{n+1} \Delta \bar{\epsilon}_{n} & =\boldsymbol{\Sigma}_{n+1}: \Delta \boldsymbol{\epsilon}_{n}^{\mathrm{p}},\end{cases}
$$

where $\bar{\kappa}_{n}=\bar{\kappa}\left(f_{n}^{v}, f_{n}^{p}, f_{n}^{m}\right)$ and $\bar{\mu}_{n}=\bar{\mu}\left(f_{n}^{v}, f_{n}^{p}, f_{n}^{m}\right)$ are the elastic moduli calculated for the previous microstructure and $\boldsymbol{\Sigma}_{n+1}^{\text {elas }}=\Sigma_{n}+\left(3 \bar{\kappa}_{n} \mathbb{J}+2 \bar{\mu}_{n} \mathbb{K}\right): \Delta \boldsymbol{\epsilon}_{n}$ is the elastic predictor, that is the stress tensor at time $t_{n+1}$ resulting from the strain increment $\Delta \boldsymbol{\epsilon}_{n}$ fictitiously considered as purely elastic.

\subsection{Algorithm of solution}

The algorithm essentially consists in finding $\bar{\sigma}_{n+1}$ and $\Delta \lambda_{n}$ (see Morin et al. (2015a)). The treatment of hardening is done classically using a fixed point method: (i) assume $\bar{\sigma}_{n+1}=\bar{\sigma}_{n}$; (ii) compute $\boldsymbol{\epsilon}_{n+1}^{\mathrm{p}}, \boldsymbol{\Sigma}_{n+1}$ and $\bar{\epsilon}_{n+1}$ with this value $\bar{\sigma}_{n+1}$; (iii) deduce from Eq. $(23)_{6}$ a refined estimate of $\bar{\sigma}_{n+1}$; (iv) follow the procedure until the method converges and $\bar{\sigma}_{n+1}$ reaches a stationary value.

The problem thus consists in the determination of $\epsilon_{n+1}^{\mathrm{p}}, \boldsymbol{\Sigma}_{n+1}$ and $\bar{\epsilon}_{n+1}$, for a fixed value $\bar{\sigma}_{n+1}$. First we begin with the elastic behaviour.

Elastic behaviour If the condition $\Phi\left(\Sigma_{n+1}^{\text {elas }}, f_{n}^{v}, \bar{\sigma}_{n+1}\right) \leq 0$ is met, then the evolution is purely elastic and the final mechanical state is given by

$$
\left\{\begin{array}{l}
\boldsymbol{\Sigma}_{n+1}=\boldsymbol{\Sigma}_{n+1}^{\text {elas }} \\
\boldsymbol{\epsilon}_{n+1}^{\mathrm{p}}=\boldsymbol{\epsilon}_{n}^{\mathrm{p}}
\end{array}\right.
$$

However, if $\Phi\left(\Sigma_{n+1}^{\text {elas }}, f_{n}^{v}, \bar{\sigma}_{n+1}\right)>0$, then the elastic predictor is not plastically admissible and a plastic correction is needed to ensure that the plasticity criterion is verified.

Plastic correction Using Eqs. $(23)_{1}$ and $(23)_{3}$, the stress $\boldsymbol{\Sigma}_{n+1}$ can be written as

$$
\boldsymbol{\Sigma}_{n+1}=\boldsymbol{\Sigma}_{n+1}^{\text {elas }}-\Delta \lambda_{n}\left(\frac{3 \bar{\kappa}_{n} q_{1} q_{2} f_{n}^{v}}{\bar{\sigma}_{n+1}} \sinh \left(\frac{3 q_{2} \Sigma_{n+1, m}}{2 \bar{\sigma}_{n+1}}\right) \mathbf{I}+6 \bar{\mu}_{n} \frac{\boldsymbol{\Sigma}_{n+1, d}}{\bar{\sigma}_{n+1}^{2}}\right) .
$$

This equation can be split into a mean and deviatoric parts:

$$
\left\{\begin{array}{l}
\Sigma_{n+1, m}=\Sigma_{n+1, m}^{\text {elas }}-\frac{3 \Delta \lambda_{n} \bar{\kappa}_{n} q_{1} q_{2} f_{n}^{v}}{\bar{\sigma}_{n+1}} \sinh \left(\frac{3 q_{2} \Sigma_{n+1, m}}{2 \bar{\sigma}_{n+1}}\right) \\
\Sigma_{n+1, d}=\frac{\Sigma_{n+1, d}^{\text {elas }}}{1+6 \Delta \lambda_{n} \frac{\bar{\mu}_{n}}{\bar{\sigma}_{n+1}^{2}}},
\end{array}\right.
$$


where $\boldsymbol{\Sigma}_{n+1, d}=\boldsymbol{\Sigma}_{n+1}-\frac{1}{3} \operatorname{tr}\left(\boldsymbol{\Sigma}_{n+1}\right) \mathbf{I}$ is the deviatoric part of the stress $\boldsymbol{\Sigma}_{n+1}$. By taking the von Mises norm of Eq. (26) 2 , one finally gets

$$
\left\{\begin{array}{l}
\Sigma_{n+1, m}=\Sigma_{n+1, m}^{\text {elas }}-\frac{3 \Delta \lambda_{n} \bar{\kappa}_{n} q_{1} q_{2} f_{n}^{v}}{\bar{\sigma}_{n+1}} \sinh \left(\frac{3 q_{2} \Sigma_{n+1, m}}{2 \bar{\sigma}_{n+1}}\right) \\
\Sigma_{n+1, e q}=\frac{\Sigma_{n+1, \text { eq }}^{\text {elas }}}{1+6 \Delta \lambda_{n} \frac{\bar{\mu}_{n}}{\bar{\sigma}_{n+1}^{2}}} .
\end{array}\right.
$$

The final stress $\boldsymbol{\Sigma}_{n+1}$ must lie on the yield locus which means that the mean and equivalent stresses $\Sigma_{n+1, m}$ and $\Sigma_{n+1, e q}$ must satisfy the criterion (8):

$$
\frac{\Sigma_{n+1, e q}^{2}}{\bar{\sigma}_{n+1}^{2}}+2 q_{1} f_{n}^{v} \cosh \left(\frac{3 q_{2} \Sigma_{n+1, m}}{2 \bar{\sigma}_{n+1}}\right)-1-q_{3}\left(f_{n}^{v}\right)^{2}=0
$$

Using Eqs. (26) 1 and (28), one can write the mean stress $\Sigma_{n+1, m}$ in terms of the equivalent stress $\Sigma_{n+1, e q}$

$$
\Sigma_{n+1, m}=\operatorname{sgn}\left(\Sigma_{n+1, m}^{\text {elas }}\right) \frac{2 \bar{\sigma}_{n+1}}{3 q_{2}} \arg \cosh \left(\frac{1+q_{3}\left(f_{n}^{v}\right)^{2}-\frac{\Sigma_{n+1, e q}^{2}}{\bar{\sigma}_{n+1}^{2}}}{2 q_{1} f_{n}^{v}}\right),
$$

where $\operatorname{sgn}(x)$ denotes the sign of $x$. Combining Eq. (27) and (29), one finally gets

$$
F\left(\Delta \lambda_{n}\right)=\frac{2 \bar{\sigma}_{n+1}}{3 q_{2}} \arg \cosh \left(g\left(\Delta \lambda_{n}\right)\right)+\frac{3 \Delta \lambda_{n} \bar{\kappa}_{n} q_{1} q_{2} f_{n}^{v}}{\bar{\sigma}_{n+1}} \sqrt{g\left(\Delta \lambda_{n}\right)^{2}-1}-\left|\Sigma_{n+1, m}^{\text {elas }}\right|=0,
$$

where

$$
g\left(\Delta \lambda_{n}\right)=\frac{1+q_{3}\left(f_{n}^{v}\right)^{2}-\left(\frac{\Sigma_{n+1, e q}^{\text {elas }}}{\bar{\sigma}_{n+1}+\frac{6 \Delta \lambda_{n} \bar{\mu}_{n}}{\bar{\sigma}_{n+1}}}\right)^{2}}{2 q_{1} f_{n}^{v}} .
$$

Eq. (30) is a nonlinear equation with the sole unknown $\Delta \lambda_{n}$ which may be quite easily solved using a Newton method. Once $\Delta \lambda_{n}$ is known, the stress $\boldsymbol{\Sigma}_{n+1}$ is deduced using Eqs. (25) and (26), and the increment of plastic strain $\Delta \boldsymbol{\epsilon}_{n}^{\mathrm{p}}$ is deduced using Eq. $(23)_{3}$.

\subsection{Update of internal parameters}

As explained in Section 3.1, the volume fractions of the three phases are updated using an explicit scheme (at the very end of global elastoplastic iterations, once $\epsilon_{n+1}^{\mathrm{p}}, \boldsymbol{\Sigma}_{n+1}$ and $\bar{\epsilon}_{n+1}$ have reached their stationary values):

$$
\left\{\begin{array}{l}
f_{n+1}^{v}=f_{n}^{v}+\Delta f_{n}^{\text {growth }}+\Delta f_{n}^{\text {nucleation }} \\
f_{n+1}^{p}=f_{n}^{p}-\theta \Delta f_{n}^{\text {nucleation }} \\
f_{n+1}^{m}=f_{n}^{m}-\Delta f_{n}^{\text {growth }}-(1-\theta) \Delta f_{n}^{\text {nucleation }}
\end{array}\right.
$$

where the increment of volume fraction due to growth $\Delta f_{n}^{\text {growth }}$ and nucleation $\Delta f_{n}^{\text {nucleation }}$ are given by

$$
\left\{\begin{aligned}
\Delta f_{n}^{\text {growth }} & =\left(1-f_{n}^{v}\right) \operatorname{tr} \Delta \boldsymbol{\epsilon}_{n}^{\mathrm{p}} \\
\Delta f_{n}^{\text {nucleation }} & =\frac{f_{N}}{s_{N} \sqrt{2 \pi}} \exp \left[-\frac{1}{2}\left(\frac{\bar{\epsilon}_{n+1}-\epsilon_{N}}{s_{N}}\right)^{2}\right] \times \Delta \bar{\epsilon}_{n} .
\end{aligned}\right.
$$

\section{Application to the prediction of stiffness loss during plastic deformation}

\subsection{Generalities}

Type of simulations The model's predictions are now investigated in problems involving loss of stiffness. Experimental tension tests results from the literature are considered for several heterogeneous materials including Al-SiC composites, cast irons and $\mathrm{Fe}-\mathrm{TiB}_{2}$ composites. We focus here on stiffness loss from low to moderate strain levels prior to the final failure, which are representative of processing loading paths. In that case, the experimental stress state is homogeneous within 
the test specimen. The model predictions can thus be investigated with a single (homogeneous) element subjected to an axisymmetric proportional loading with major axial stress $\Sigma_{33}$, under conditions of fixed stress triaxiality $T=\Sigma_{m} / \Sigma_{\text {eq }}=1 / 3$ (since tensile tests are considered). The non-zero components of the macroscopic stress tensor are supposed to be

$$
\Sigma_{11}=\Sigma_{22}=\Sigma_{m}-\frac{1}{3} \Sigma_{e q}, \quad \Sigma_{33}=\Sigma_{m}+\frac{2}{3} \Sigma_{e q} .
$$

Two quantities will be studied: (i) the evolution of the axial stress $\Sigma_{33}$ versus the axial strain $\varepsilon_{33}$ (the stress-strain curve) and (ii) the evolution of the macroscopic Young's modulus $\bar{E}$ versus the axial strain $\varepsilon_{33}$ (the loss of stiffness).

Calibration of the model's parameters In the following applications, a classical power-law isotropic hardening is assumed:

$$
\sigma(\epsilon)=\sigma_{y}\left(1+\frac{\epsilon}{\epsilon_{0}}\right)^{n}
$$

where $\sigma_{y}$ is the initial yield stress of sound composite, $\epsilon_{0}$ is the hardening parameter and $n$ is the hardening exponent.

The complete elastic-plastic model including damage requires the determination of 14 parameters:

- The initial volume fractions of the particles, matrix and voids $\left(f_{0}^{p}, f_{0}^{m}, f_{0}^{v}\right)$ can be (classically) calibrated using microstructural image analyses from SEM-EBSD or X-ray tomography.

- The elastic properties of the matrix and the particles $\left(\kappa_{p}, \mu_{p}, \kappa_{m}, \mu_{m}\right)$ or equivalently $\left(E_{p}, v_{p}, E_{m}, v_{m}\right)$, can be evaluated separately on homogeneous constituents using experimental methods (mechanical tensile tests, indentation) or numerical methods (ab initio calculations).

- The plastic properties $\left(\sigma_{y}, \epsilon_{0}, n\right)$ can be determined with a mechanical tensile test.

- The determination of nucleation parameters $\left(\epsilon_{N}, s_{N}, f_{N}, \theta\right)$ requires more attention since the model is heuristic. First, the fraction of void nucleating particles $f_{N}$ can be identified as the initial volume fraction of particles $f_{0}^{p}$. In the case of matrix/particles interface decohesion, this assumption is reasonable since all of the interfaces will eventually break. In the case of particle cracking, this assumption is probably less true but it should still be a good estimate of the order of magnitude of the fraction of void nucleating particles. The average nucleation strain $\epsilon_{N}$ can be estimated precisely from microscopic image analyses using in-situ tensile test measurements with SEM or tomography. In absence of microscopic measurements, it could also be evaluated as a percentage of the strain-to-fracture of the material in tension. The standard deviation $s_{N}$ is an adjustable parameter of the model; in this work a constant value $s_{N}=0.045$ has been considered in all simulations. Finally, the order of magnitude of the parameter $\theta$, which is related to the type of damage mechanism, can be estimated with in-situ analyses or ex-situ observations of interrupted tensile tests just before fracture.

Since the simulations are performed for low to moderate strain levels prior to the final failure, Tvergaard (1981)'s parameters $q_{1}, q_{2}$ and $q_{3}$ have little influence on the plastic behavior. The values $q_{1}=q_{2}=q_{3}=1$ are thus considered in all the numerical simulations.

\subsection{Case of Al-SiC composites}

We first consider the case of Al-SiC composites, which constitutes one of the most common and available metal-matrix composites due to low production costs. This class of materials is notably used in aeronautics due to an increase of several mechanical properties such as the toughness, the hardness and the elastic modulus, among others (Ozben et al., 2008). Experimental studies have shown that damage in Al-SiC composites is mainly due to the cracking of SiC particles (Buffiere et al., 1997; Derrien et al., 1999; Williams et al., 2010).

In order to study quantitatively the predictions of the model developed, we focus on the experimental results of Derrien et al. (1999) which provide the macroscopic stress-strain curves as well as the loss of stiffness in several Al-SiC composites. Two Al-SiC composites are considered, one with a volume fraction of particles of $15 \%$ and the other with a volume fraction of particles of $20 \%$. The model parameters are calibrated using the procedure described in Section 4.1. Thus, the values $f_{0}^{m}=0.85, f_{0}^{p}=0.15$ and $f_{0}^{v}=0$ are considered in the first case while the values $f_{0}^{m}=0.8, f_{0}^{p}=0.2$ and $f_{0}^{v}=0$ are considered in the second case. Microstructure analyses show that the shape of the SiC particles is in general spherical (Derrien et al., 1999). The values for the elastic constants are as follows: $E_{m}=76.5 \mathrm{GPa}$ and $v=0.3$ are considered for the aluminum matrix (see Derrien et al. (1999)) and $E_{p}=492 \mathrm{GPa}$ and $v_{p}=0.21$ are considered for the SiC particles which are consistent with values of the literature (Snead et al., 2007). The plastic properties $\sigma_{y}, \epsilon_{0}$ and $n$ are given in Tables 1 and 2. (It should be noted that the value of the initial yield stress $\sigma_{y}$ is slightly higher in the case $f_{0}^{p}=0.2$; this is reasonable

Table 1

\begin{tabular}{|c|c|c|c|c|c|c|c|c|c|}
\hline \multicolumn{4}{|c|}{ Elastic properties } & \multicolumn{3}{|c|}{ Plastic properties } & \multicolumn{3}{|c|}{ Nucleation parameters } \\
\hline$E_{p}[\mathrm{GPa}]$ & $v_{p}$ & $E_{m}[\mathrm{GPa}]$ & $v_{m}$ & $\sigma_{y}[\mathrm{MPa}]$ & $\epsilon_{0}$ & $n$ & $\epsilon_{N}$ & $s_{N}$ & $f_{N}$ \\
\hline 492 & 0.21 & 76.5 & 0.33 & 260 & 0.00033 & 0.15 & 0.04 & 0.045 & 0.15 \\
\hline
\end{tabular}

Parameters considered in the case of $15 \%-\mathrm{SiC} \mathrm{Al-SiC} \mathrm{composite.}$ 
Table 2

Parameters considered in the case of $20 \%-\mathrm{SiC}$ Al-SiC composite.

\begin{tabular}{|c|c|c|c|c|c|c|c|c|c|}
\hline \multicolumn{4}{|c|}{ Elastic properties } & \multicolumn{3}{|c|}{ Plastic properties } & \multicolumn{3}{|c|}{ Nucleation parameters } \\
\hline$E_{p}[\mathrm{GPa}]$ & $v_{p}$ & $E_{m}[\mathrm{GPa}]$ & $v_{m}$ & $\sigma_{y}[\mathrm{MPa}]$ & $\epsilon_{0}$ & $n$ & $\epsilon_{N}$ & $s_{N}$ & $f_{N}$ \\
\hline 492 & 0.21 & 76.5 & 0.33 & 280 & 0.00033 & 0.15 & 0.015 & 0.045 & 0.20 \\
\hline
\end{tabular}

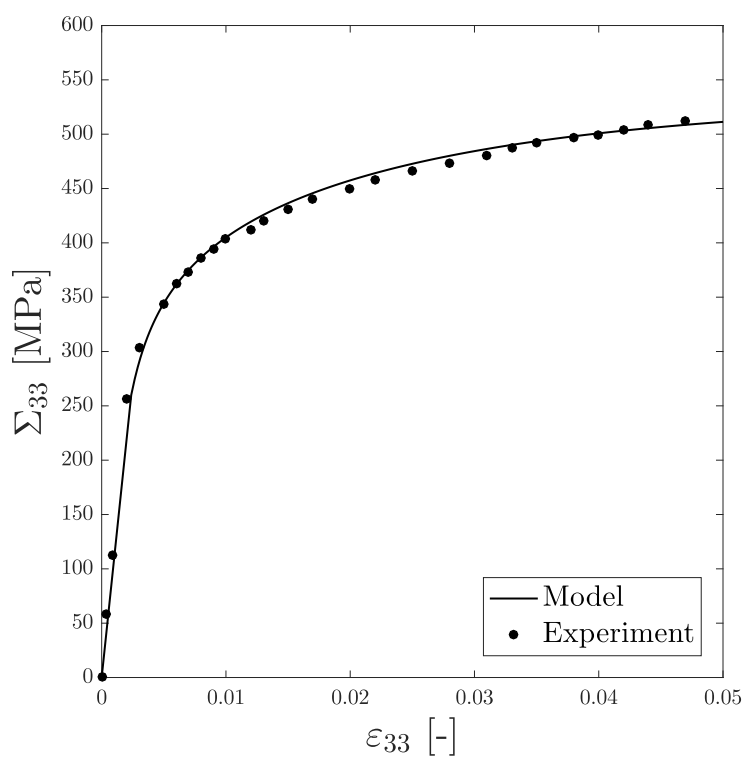

(a)

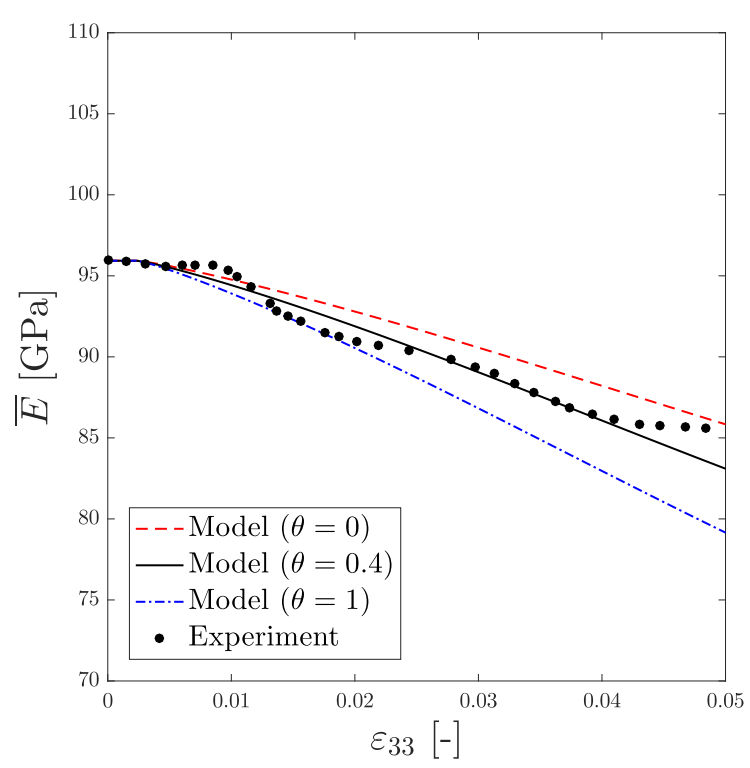

(b)

Fig. 3. Comparisons between experimental and model's predictions in the case of a 15\%-SiC Al-SiC composite: (a) stress-strain curves and (b) stiffness loss.

because the consequence of an increase of the volume fraction of particles $f^{p}$ is an unloading of the matrix which consequently requires a higher macroscopic stress to promote plastic yielding of the matrix). Finally, the nucleation parameters are taken as follows: $f_{N}=f_{0}^{p}=[0.15 ; 0.2], s_{N}=0.045$ and $\epsilon_{N}=[0.04 ; 0.015]$. This corresponds respectively, for the nucleation strains, to a percentage of the strain-to-fracture of about 0.4 and 0.6 . A summary of all the parameters used is given in Tables 1 and 2, in the cases of a 15\% and 20\% of SiC, respectively.

The stress-strain curves and the evolution of the elastic modulus are provided in Figs. 3 and 4, in the cases of a 15\% and $20 \%$ of $\mathrm{SiC}$, respectively. It should be noted that the stress-strain curves are shown only for $\theta=0.4$. The cases $\theta=0$ and $\theta=1$ are not represented because all the curves would be indistinguishable. Indeed, in the model developed, the parameter $\theta$ only modifies the elastic behavior after some plastic deformation: the initial elastic slope and the plastic behavior are not affected by $\theta$.

Overall, the experimental results are well reproduced by the model in the two cases considered. Some comments are in order:

- The initial value for Young's modulus is well predicted by the model for the two materials (15\% and $20 \%$ of $\mathrm{SiC}$ ). The mean-field homogenization scheme considered, which depends only on the values of elastic constants of the phases and their volume fractions, is thus relevant to describe the microstructure.

- The loss of stiffness due to plastic deformation is also well reproduced by the model for the same value $\theta=0.4$ in the two cases considered ( $15 \%$ and $20 \%$ of $\mathrm{SiC}$ ). This suggests that (i) the damage mechanism is the same for the two materials, irrespective of the volume fraction of particles and (ii) damage is mainly due to cracking and second-phase nucleation since $\theta$ is closer to 0 than 1 , which is consistent with the experimental observations where no decohesion was observed (Derrien et al., 1999).

- The lower value for $\epsilon_{N}$ in the second case (with $20 \%$ of particles) is due to the fact that cracking arises more quickly in that case, due to a higher local stress state (Derrien et al., 1999). This is also consistent with the fact that the strain-to-fracture is lower in that case.

- In the model predictions, it is interesting to note that the value $\theta=1$ leads to a greater degradation than the value $\theta=0$. This behavior is expected in the case of particles that play the role of reinforcements (which is the case here) since the decohesion of the matrix/particles interfaces (which corresponds to the case $\theta=1$ ) would lead to a greater loss of stiffness than the nucleation of second-phase particles (which corresponds to the case $\theta=0$ ), because replac- 


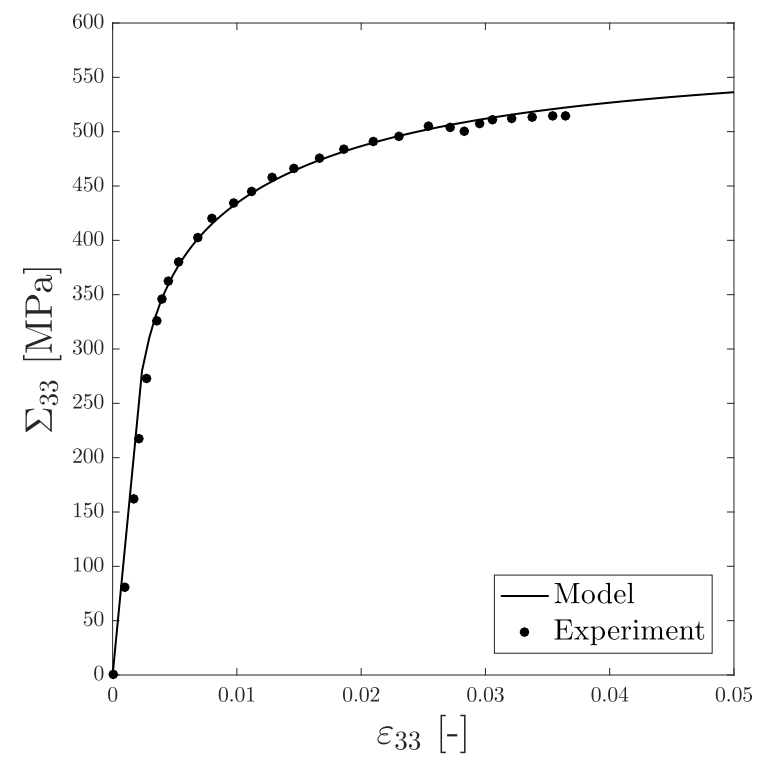

(a)

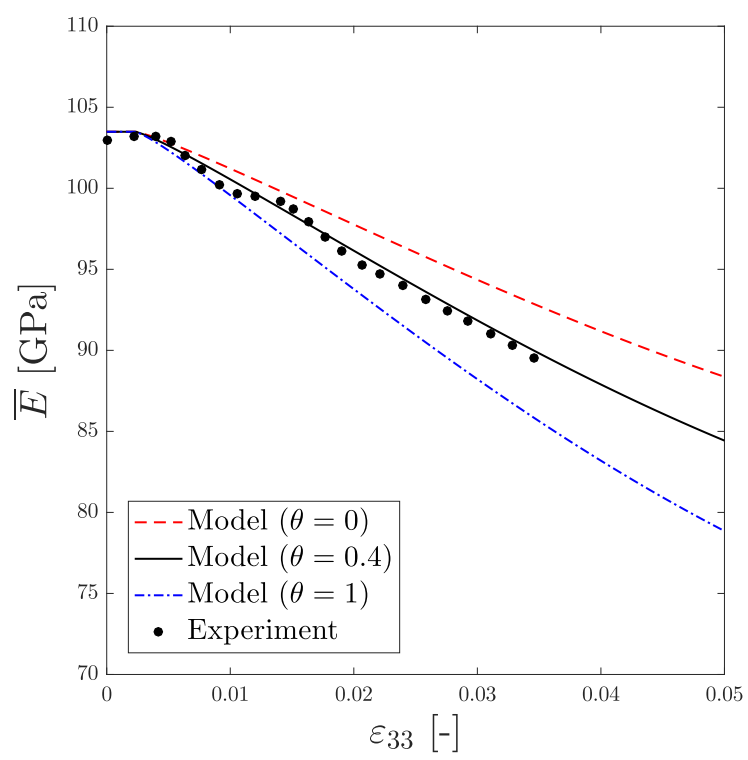

(b)

Fig. 4. Comparisons between experimental and model's predictions in the case of a 20\%-SiC Al-SiC composite: (a) stress-strain curves and (b) stiffness loss.

ing particles with voids is more severe than replacing the matrix with voids. It is worth noting that the extreme values $\theta=0$ and $\theta=1$ almost define an envelope containing the experimental loss of stiffness.

- After a deformation of about 0.05 , the difference of stiffness loss predicted by the model between the values $\theta=0$ and $\theta=1$ is respectively of about $5 \mathrm{GPa}$ and $10 \mathrm{GPa}$ in the cases of a $15 \%-\mathrm{SiC}$ and $20 \%-\mathrm{SiC}$ Al-SiC composites. This trend is expected since the parameter $\theta$ has more impact when a higher volume fraction of particles is considered.

- It is worth noting that the experimental stiffness shows a non-linear evolution, which may be due to the occurrence of different nucleation mechanisms at different strains. These non-linearities, which are not reproduced by the model, may be captured by adding a strain dependency to the parameter $\theta$ or by using a more sophisticated nucleation model.

\subsection{Case of a nodular cast iron}

We consider the case of nodular cast irons, which are materials of interest in industrial applications due to a good compromise between physical properties and manufacturing costs (Hütter et al., 2015). These materials are strictly speaking carbon-rich iron alloys but they may also be seen as metal-matrix composites due to the presence of graphite particles in a moderate volume fraction (typically 7-15\%). The particles of graphite do not play the role of classical reinforcements since they are softer than the ferritic matrix. Experimental studies have shown that damage in cast irons is mainly due to the decohesion of the matrix/graphite particles interfaces (Berdin et al., 2001; Dong et al., 1997; Hütter et al., 2015; Murakami et al., 1998; Tomičević et al., 2016).

The model's predictions are compared with the experimental results of Murakami et al. (1998) which provide the macroscopic strain-stress curve and the evolution of the macroscopic Young's modulus in a JIS FCD400 spheroidized graphite cast iron; this material consists in a ferritic matrix containing graphite particles. A metallographical microstructure of the cast iron has been analyzed and reveals that the graphite particles are spherical and in a volume fraction of about $15 \%$. Initially we thus consider the values $f_{0}^{m}=0.85, f_{0}^{p}=0.15$ and $f_{0}^{v}=0$ for the volume fractions of the phases. The elastic constants considered are as follows: $E_{m}=210 \mathrm{GPa}$ and $v=0.3$ are classically considered for the ferritic matrix and $E_{p}=30 \mathrm{GPa}$ and $v_{p}=0.3$ are considered for the graphite particles which are in agreement with the literature (Carazo et al., 2014). The plastic properties $\sigma_{y}, \epsilon_{0}$ and $n$ are given in Table 3. Finally, the nucleation parameters are as follows: $f_{N}=f_{0}^{p}=0.15, s_{N}=0.045$

Table 3

Parameters considered in the case of a cast iron (Murakami et al., 1998).

\begin{tabular}{|c|c|c|c|c|c|c|c|c|c|}
\hline \multicolumn{4}{|c|}{ Elastic properties } & \multicolumn{3}{|c|}{ Plastic properties } & \multicolumn{3}{|c|}{ Nucleation parameters } \\
\hline$E_{p}[\mathrm{GPa}]$ & $v_{p}$ & $E_{m}[\mathrm{GPa}]$ & $v_{m}$ & $\sigma_{y}[\mathrm{MPa}]$ & $\epsilon_{0}$ & $n$ & $\epsilon_{N}$ & $s_{N}$ & $f_{N}$ \\
\hline 30 & 0.3 & 210 & 0.3 & 220 & 0.0013 & 0.195 & 0.02 & 0.045 & 0.15 \\
\hline
\end{tabular}




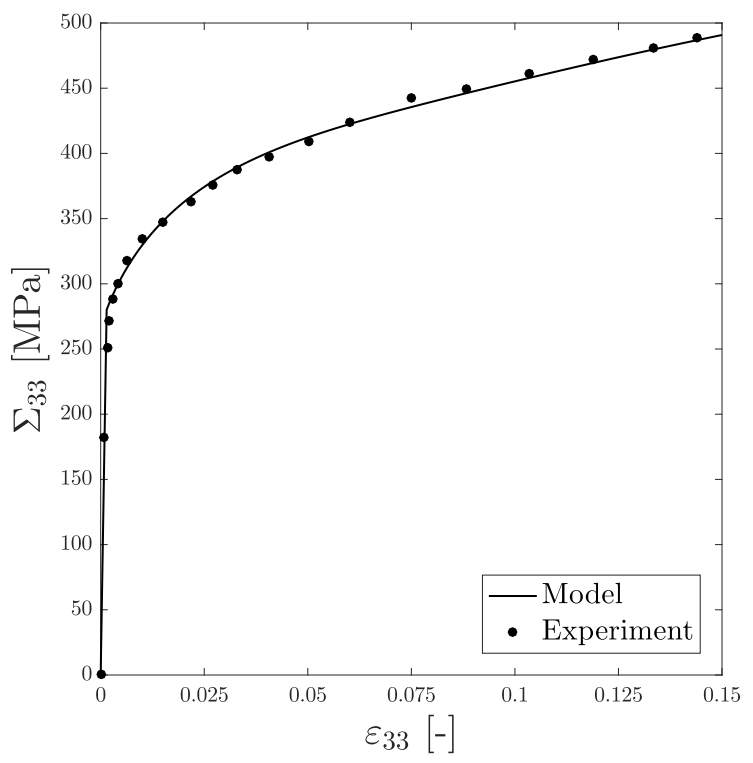

(a)

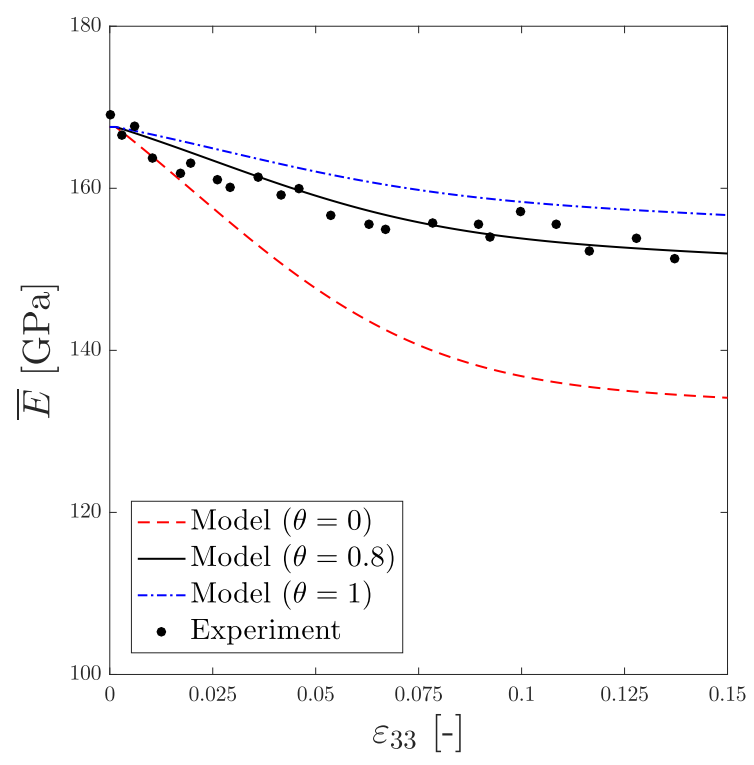

(b)

Fig. 5. Comparisons between experimental and model's predictions in the case of a nodular cast iron: (a) stress-strain curves and (b) stiffness loss.

and $\epsilon_{N}=0.02$. This corresponds, for the nucleation strain, to a percentage of the strain-to-fracture of about 0.1 . A summary of all the parameters used is given in Table 3.

The stress-strain curves and the evolution of the elastic modulus are provided in Fig. 5. It should be noted that the stress-strain curves are shown only for $\theta=0.8$.

Overall, the experimental results are well reproduced by the model. Some comments are in order:

- The initial value for Young's modulus is again well predicted by the homogenization scheme.

- The loss of stiffness due to plastic deformation is also well reproduced by the model for a value $\theta=0.8$. This value of $\theta$ suggests that the damage mechanism in cast irons is mostly due to the decohesion of the matrix/particles interfaces, which is consistent with the experimental observations. Indeed in cast irons, this interface is not very cohesive which implies that (i) the value of $\theta$ in the model is closer to 1 than 0 and (ii) the value of $\epsilon_{N}$ is low.

- In the model predictions, it is interesting to note that the value $\theta=0$ leads to more damage than the value $\theta=1$. In the case of cast irons, this behavior is expected since the particles do not play the role of reinforcements. Again, it is worth noting that the extremal values $\theta=0$ and $\theta=1$ almost define an envelope containing the experimental loss of stiffness.

\subsection{Case of $\mathrm{Fe}-\mathrm{TiB}_{2}$ composites}

We finally consider the case of $\mathrm{Fe}-\mathrm{TiB}_{2}$ composites which are materials of interest in structural lightweight applications. Experimental observations showed that damage is mainly caused by the cracking of $\mathrm{TiB}_{2}$ particles (Gaspérini et al., 2017; Hadjem-Hamouche et al., 2012, 2018).

We consider the results of Hadjem-Hamouche et al., 2018 which include the macroscopic stress-strain curves and the initial value of Young's modulus. The loss of stiffness is not provided in the literature, so the predictions of the model will thus permit only to estimate the possible decrease in macroscopic Young's modulus. The composite considered contains about $13 \%$ of particles (Hadjem-Hamouche et al., 2018), so initially we consider the values $f_{0}^{m}=0.87, f_{0}^{p}=0.13$ and $f_{0}^{v}=$ 0 for the volume fractions of the phases. The elastic constants considered are as follows: $E_{m}=210$ GPa and $v=0.3$ are considered for the ferritic matrix and $E_{p}=583 \mathrm{GPa}$ and $v_{p}=0.11$ are considered for the $\mathrm{TiB}_{2}$ particles (Hadjem-Hamouche et al., 2018). The plastic properties $\sigma_{y}, \epsilon_{0}$ and $n$ are given in Table 4. Finally, the nucleation parameters are taken as follows:

Table 4

Parameters considered in the case of a $\mathrm{Fe}^{-\mathrm{TiB}_{2}}$ composite (Hadjem-Hamouche et al., 2018).

\begin{tabular}{|c|c|c|c|c|c|c|c|c|c|}
\hline \multicolumn{4}{|c|}{ Elastic properties } & \multicolumn{3}{|c|}{ Plastic properties } & \multicolumn{3}{|c|}{ Nucleation parameters } \\
\hline$E_{p}[\mathrm{GPa}]$ & $v_{p}$ & $E_{m}[\mathrm{GPa}]$ & $v_{m}$ & $\sigma_{y}[\mathrm{MPa}]$ & $\epsilon_{0}$ & $n$ & $\epsilon_{N}$ & $s_{N}$ & $f_{N}$ \\
\hline 583 & 0.11 & 210 & 0.3 & 200 & 0.0007 & 0.22 & 0.1 & 0.045 & 0.13 \\
\hline
\end{tabular}




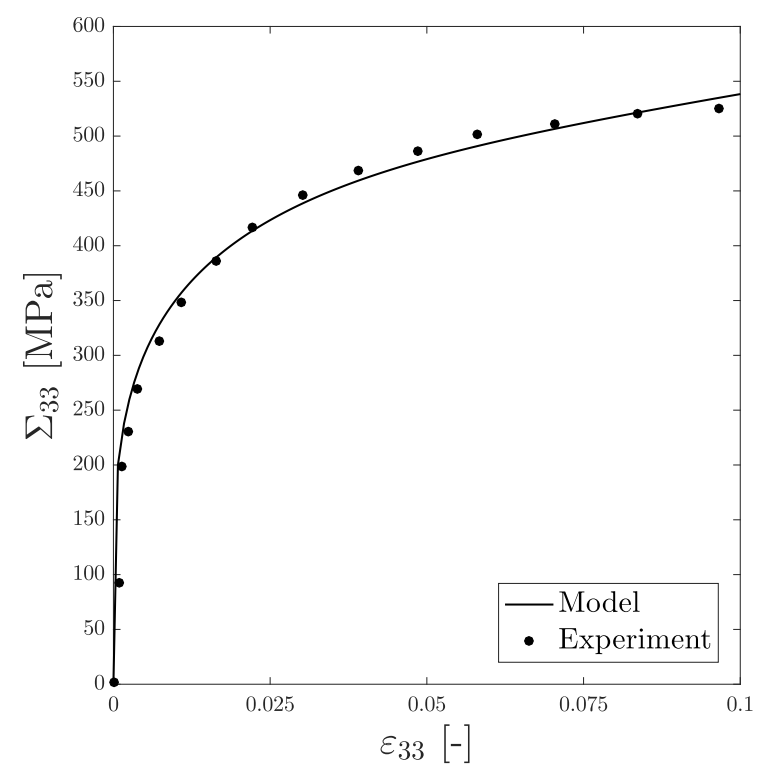

(a)

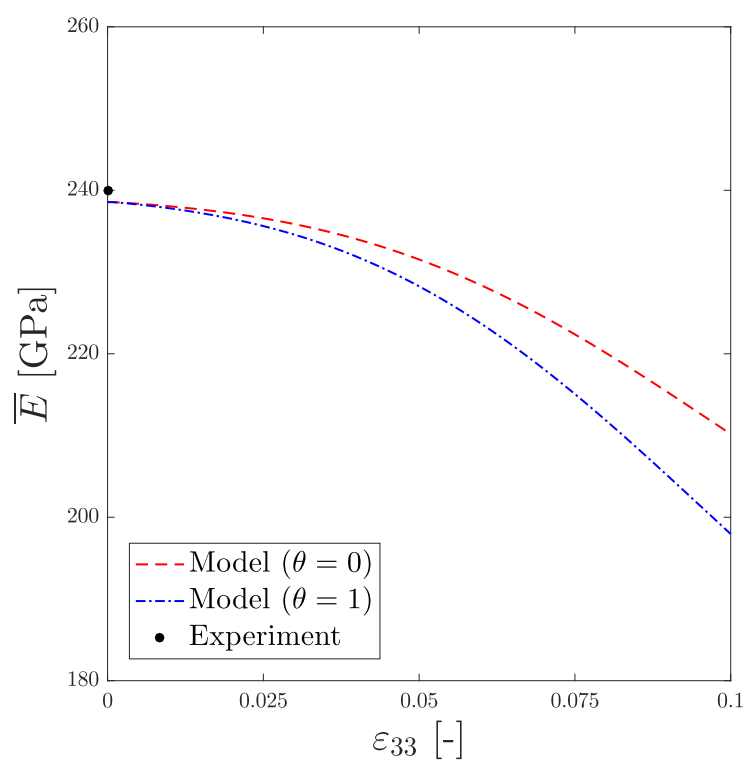

(b)

Fig. 6. Comparisons between experimental and model's predictions in the case of a Fe-TiB ${ }_{2}$ composite: (a) stress-strain curves and (b) stiffness loss.

$f_{N}=f_{0}^{p}=0.13, s_{N}=0.045$ and $\epsilon_{N}=0.1$. This corresponds, for the nucleation strain, to a percentage of the strain-to-fracture of about 0.5 . A summary of all the parameters used is given in Table 4 .

The stress-strain curves and the evolution of the elastic modulus are provided in Fig. 6. It should be noted that the stress-strain curves are shown only for $\theta=0.5$.

Again, the initial value for the macroscopic Young's modulus is again well predicted by the homogenization scheme. A stiffness loss between 7 and $10 \mathrm{GPa}$ is estimated by the model after a deformation of about $5 \%$, using a set of nucleation parameters that seem physically relevant where the nucleation strain was taken as half of the strain-to-fracture.

\section{Conclusion}

The aim of this paper was to develop a homogenized model that is able to describe the progressive loss of stiffness in heterogeneous metal-matrix composites.

An approximate homogenization model was derived in Section 2 by combining a mean-field homogenized scheme for elastic properties with a Gurson-type model for plastic properties. The microstructure of the metal-matrix composite is accounted for through the volume fractions of three phases (matrix, particles and voids) which evolve during plastic straining. A heuristic parameter is introduced in the void nucleation law which permits to distinguish between several nucleation mechanisms. Thus, volume fractions of both matrix and particles can be modified due to plastic flow driven by Gurson's potential, which ultimately leads to a modification of the macroscopic elastic properties. The complete model was then numerically implemented in Section 3. The internal parameters that describe the microstructure are discretized with an explicit scheme which allows the model to fall into the class of "generalized standard" materials. In Section 4 the model was finally applied to the prediction of stiffness loss in several materials (Al-SiC composites, cast irons and $\mathrm{Fe}-\mathrm{TiB}_{2}$ ). In all cases, the model, calibrated with physical values of internal parameters, is in very good agreement with experimental data of the literature and permits to predict the influence of progressive damage on elastic modulus.

The present work has shown that the problem of stiffness loss can be qualitatively reproduced with a simplified homogenized model. However, some further developments are needed in the following directions:

- The prediction of stiffness loss was investigated only on two materials (Al-SiC composites and cast irons). It is necessary to extend the comparisons with experimental data on several other materials. Experimental investigations of stiffness loss on $\mathrm{Fe}^{-\mathrm{TiB}} \mathrm{B}_{2}$ are in progress.

- Particles were supposed to be spherical and elastically isotropic. The extension of this work to a more general situation with anisotropic elasticity and shape effects of the particles, in order to account for fiber-reinforced composites for instance, is straightforward in elasticity within the mean-field homogenization framework considered. However, difficulties may arise to model the plastic behavior:

- Nucleated voids may have several shapes: ellipsoidal or cylindrical shapes due to the primary particles and spherical shapes due to the second-phase particles. The use of a more sophisticated void growth model including several populations of cavities appears necessary in that case (Shen et al., 2017; Vincent et al., 2014). 
- The induced elastic anisotropy will modify the onset of plasticity and could invalidate the hypothesis of an isotropic von Mises plastic behavior for the sound composite. This can be addressed by using incremental homogenization (Agoras et al., 2016; Brassart et al., 2011; Doghri et al., 2011; Doghri and Ouaar, 2003; Lahellec and Suquet, 2007) that can allow the determination of the effective stress strain relation. In the case of anisotropic plastic yielding, it would be necessary to enrich the damage model considered by using an extension of Gurson's approach accounting for anisotropy of the matrix (Benzerga and Besson, 2001; Monchiet et al., 2008; Morin et al., 2015b).

- The damage model derived in this work suffers inevitably from the discrepancies of Gurson's model in the case of low stress triaxiality loadings. The use of recent improvements of Gurson's model (Madou and Leblond, 2012; Morin et al., 2016) that account for void shape effects should permit to address the prediction of stiffness loss in presence of shear-dominated loadings.

- The effect of material length scale has not been considered in this work. In the case of nanosized particles and voids, size effects can arise due to gradient effects and may increase the yield strength of the material (Legarth and Niordson, 2010; Niordson and Tvergaard, 2001). Thus, in the case of metal-matrix nanocomposites, it could be interesting to extend the present work by considering micromechanical models that include size effects for elasticity (Brisard et al., 2010) and porous plasticity (Dormieux and Kondo, 2010; Niordson and Tvergaard, 2019), in order to investigate the effect of length scale on stiffness loss.

\section{Declaration of Competing Interest}

None.

\section{Acknowledgments}

Fruitful discussions with S. Berbenni, F. Bonnet, J. Gene and N. Gey are gratefully acknowledged. This work is supported by the $\mathrm{C}$

\section{References}

Agoras, M., Avazmohammadi, R., Ponte Castañeda, P. 2016. Incremental variational procedure for elasto-viscoplastic composites and application to polymer-

and metal-matrix composites reinforced by spheroidal elastic particles. Int. J. Solids Struct. 97-98, 668-686.

Babout, L., Maire, E., Buffiere, J.Y., Fougeres, R., 2001. Characterization by X-ray computed tomography of decohesion, porosity growth and

coalescence in

model metal matrix composites. Acta Mater. 49, 2055-2063.

Babout, L., Maire, E., Fougeres, R., 2004. Damage initiation in model metallic materials: X-ray tomography and modelling. Acta Mater. 52, $2475-2487$.

Benzerga, A.A., Besson, J., 2001. Plastic potentials for anisotropic porous solids. Eur. J. Mech. - A/Solids 20, 397-434.

Benzerga, A.A., Leblond, J.B., 2010. Ductile fracture by void growth to coalescence. Adv. Appl. Mech. 44, 169-305.

Benzerga, A.A., Leblond, J.B., Needleman, A., Tvergaard, V., 2016. Ductile failure modeling. Int. J. Fract. 201, 29-80.

Berdin, C., Dong, M.J., Prioul, C., 2001. Local approach of damage and fracture toughness for nodular cast iron. Eng. Fract. Mech. 68, $1107-1117$.

Brassart, L., Stainier, L., Doghri, I., Delannay, L., 2011. A variational formulation for the incremental homogenization of elasto-plastic composites. J. Mech Phys. Solids 59, 2455-2475.

Brechet, Y., Embury, J.D., Tao, S., Luo, L., 1991. Damage initiation in metal matrix composites. Acta Metallurgica et Materialia 39, 1781-1786.

Brisard, S., Dormieux, L., Kondo, D., 2010. Hashin Shtrikman bounds on the bulk modulus of a nanocomposite with spherical inclusions and interface effects. Comput. Mater. Sci. 48, 589-596.

Buffiere, J.Y., Maire, E., Verdu, C., Cloetens, P., Pateyron, M., Peix, G., Baruchel, J., 1997. Damage assessment in an Al/SiC composite during monotonic tensile tests using synchrotron X-ray microtomography. Mater. Sci. Eng. A 234-236, 633-635.

Carazo, F.D., Giusti, S.M., Boccardo, A.D., Godoy, L.A., 2014. Effective properties of nodular cast-iron: a multi-scale computational approach. Comput. Mater. Sci. 82, 378-390.

Chawla, N., Chawla, K.K., 2006. Microstructure-based modeling of the deformation behavior of particle reinforced metal matrix composites. J. Mater. Sci. 41, 913-925.

Christman, T., Needleman, A., Suresh, S., 1989. An experimental and numerical study of deformation in metal-ceramic composites. Acta Metall. 37, 3029-3050.

Chu, C.C., Needleman, A., 1980. Void nucleation effects in biaxially stretched sheets. J. Eng. Mater. Technol. 102, 249-256.

Derrien, K., Baptiste, D., Guedra-Degeorges, D., Foulquier, J., 1999. Multiscale modeling of the damaged plastic behavior and failure of Al/SiCp composites.

Int. J. Plast. 15, 667-685.

Doghri, I., Brassart, L., Adam, L., Gerard, J.S., 2011. A second-moment incremental formulation for the mean-field homogenization of elasto-plastic composites. Int. J. Plast. 27, 352-371.

Doghri, I., Ouaar, A., 2003. Homogenization of two-phase elasto-plastic composite materials and structures: study of tangent operators, cyclic plasticity and numerical algorithms. Int. J. Solids Struct. 40, 1681-1712.

Dong, M.J., Prioul, C., François, D., 1997. Damage effect on the fracture toughness of nodular cast iron: part i. Damage characterization and plastic flow stress modeling. Metall. Mater. Trans. A 28, 2245-2254.

Dormieux, L., Kondo, D., 2010. An extension of Gurson model incorporating interface stresses effects. Int. J. Eng. Sci. 48, 575-581.

Enakoutsa, K., Leblond, J., Perrin, G., 2007. Numerical implementation and assessment of a phenomenological nonlocal model of ductile rupture. Comput. Methods Appl. Mech.Eng. 196, 1946-1957.

Gaspérini, M., Dammak, M., Franciosi, P., 2017. Stress estimates for particle damage in Fe-TiB2 metal matrix composites from experimental data and simulation. Eur. J. Mech. - A/Solids 64, 85-98. 
Ghosh, S., Moorthy, S., 1998. Particle fracture simulation in non-uniform microstructures of metal matrix composites. Acta Mater. 46, 965-982.

González, C., LLorca, J., 2000. A self-consistent approach to the elasto-plastic behaviour of two-phase materials including damage. J. Mech. Phys. Solids 48, $675-692$.

Gurson, A.L., 1977. Continuum theory of ductile rupture by void nucleation and growth: Part I-Yield criteria and flow rules for porous ductile media. ASME J. Eng. Mater. Technol. 99, 2-15.

Hadjem-Hamouche, Z., Chevalier, J.P., Cui, Y., Bonnet, F., 2012. Deformation behavior and damage evaluation in a new titanium diboride (TiB2) steel-based composite. Steel Res. Int. 83, 538-545.

Hadjem-Hamouche, Z., Derrien, K., Héripré, E., Chevalier, J.P., 2018. In-situ experimental and numerical studies of the damage evolution and fracture in a Fe-TiB2 composite. Mater. Sci. Eng. A 724, 594-605.

Halphen, B., Nguyen, Q.S., 1975. Sur les matériaux standard généralisés. Journal de Mécanique 14, 39-63.

Hütter, G., Zybell, L., Kuna, M., 2015. Micromechanisms of fracture in nodular cast iron: from experimental findings towards modeling strategies a review. Eng. Fract. Mech. 144, 118-141.

Ibrahim, I.A., Mohamed, F.A., Lavernia, E.J., 1991. Particulate reinforced metal matrix composites a review. J. Mater. Sci. 26, $1137-1156$.

Lahellec, N., Suquet, P., 2007. On the effective behavior of nonlinear inelastic composites: I. Incremental variational principles. J. Mech. Phys. Solids 55, $1932-1963$.

Legarth, B.N., Niordson, C.F., 2010. Debonding failure and size effects in micro-reinforced composites. Int. J. Plast. 26, 149-165.

LLorca, J., González, C., 1998. Microstructural factors controlling the strength and ductility of particle-reinforced metal-matrix composites. J. Mech. Phys. Solids 46, 1-28.

LLorca, J., Needleman, A., Suresh, S., 1991. An analysis of the effects of matrix void growth on deformation and ductility in metal-ceramic composites. Acta Metallurgica et Materialia 39, 2317-2335.

Madou, K., Leblond, J.B., 2012. A Gurson-type criterion for porous ductile solids containing arbitrary ellipsoidal voidsI: limit-analysis of some representative cell. J. Mech. Phys. Solids 60, 1020-1036.

Maire, E., Carmona, V., Courbon, J., Ludwig, W., 2007. Fast X-ray tomography and acoustic emission study of damage in metals during continuous tensile tests. Acta Mater. 55, 6806-6815.

Michel, J.C., Moulinec, H., Suquet, P., 1999. Effective properties of composite materials with periodic microstructure: a computational approach. Comput. Methods Appl. Mech.Eng. 172, 109-143.

Mishnaevsky, L., Derrien, K., Baptiste, D., 2004. Effect of microstructure of particle reinforced composites on the damage evolution: probabilistic and numerical analysis. Compos. Sci. Technol. 64, 1805-1818.

Monchiet, V., Cazacu, O., Charkaluk, E., Kondo, D., 2008. Macroscopic yield criteria for plastic anisotropic materials containing spheroidal voids. Int. J. Plast. 24, 1158-1189.

Mori, T., Tanaka, K., 1973. Average stress in matrix and average elastic energy of materials with misfitting inclusions. Acta Metall. 21, 571-574.

Morin, L., Kondo, D., Leblond, J.B., 2015. Numerical assessment, implementation and application of an extended Gurson model accounting for void size effects. Eur. J. Mech. - A/Solids 51, 183-192.

Morin, L., Leblond, J.B., Kondo, D., 2015. A Gurson-type criterion for plastically anisotropic solids containing arbitrary ellipsoidal voids. Int. J. Solids Struct. 77, 86-101.

Morin, L., Leblond, J.B., Tvergaard, V., 2016. Application of a model of plastic porous materials including void shape effects to the prediction of ductile failure under shear-dominated loadings. J. Mech. Phys. Solids 94, 148-166.

Mortensen, A., LLorca, J., 2010. Metal matrix composites. Annu. Rev. Mater. Res. 40, 243-270.

Moulinec, H., Suquet, P., 1998. A numerical method for computing the overall response of nonlinear composites with complex microstructure. Comput. Methods Appl. Mech.Eng. 157, 69-94.

Mueller, R., Mortensen, A., 2006. Simplified prediction of the monotonic uniaxial stress strain curve of non-linear particulate composites. Acta Mater. 54, 2145-2155.

Murakami, S., Hayakawa, K., Liu, Y., 1998. Damage evolution and damage surface of elastic-plastic-damage materials under multiaxial loading. Int. J. Damage Mech. 7, 103-128.

Needleman, A., 1987. A continuum model for void nucleation by inclusion debonding. J. Appl. Mech. 54, 525-531.

Niordson, C.F., Tvergaard, V., 2001. Nonlocal plasticity effects on the tensile properties of a metal matrix composite. Eur. J. Mech. - A/Solids 20, 601-613.

Niordson, C.F., Tvergaard, V., 2019. A homogenized model for size-effects in porous metals. J. Mech. Phys. Solids 123, $222-233$.

Okamoto, N.L., Kusakari, M., Tanaka, K., Inui, H., Otani, S., 2010. Anisotropic elastic constants and thermal expansivities in monocrystal CrB2, TiB2, and ZrB2. Acta Mater. 58, 76-84.

Ozben, T., Kilickap, E., Çakir, O., 2008. Investigation of mechanical and machinability properties of SiC particle reinforced Al-MMC. J. Mater. Process. Technol. $198,220-225$.

Pan, J., Saje, M., Needleman, A., 1983. Localization of deformation in rate sensitive porous plastic solids. Int. J. Fract. 21, $261-278$.

Ponte Castañeda, P., Suquet, P., 1997. Nonlinear composites. Adv. Appl. Mech. 34, 171-302.

Segurado, J., González, C., Llorca, J., 2003. A numerical investigation of the effect of particle clustering on the mechanical properties of composites. Acta Mater. 51, 2355-2369.

Segurado, J., LLorca, J., 2002. A numerical approximation to the elastic properties of sphere-reinforced composites. J. Mech. Phys. Solids 50, 2107-2121.

Shakoor, M., Bernacki, M., Bouchard, P.O., 2018. Ductile fracture of a metal matrix composite studied using 3d numerical modeling of void nucleation and coalescence. Eng. Fract. Mech. 189, 110-132.

Shen, W.Q., Shao, J.F., Kondo, D., 2017. Macroscopic criteria for Green type porous materials with spheroidal voids: application to double porous materials. Int. J. Numer. Anal. Methods Geomech. 41, 1453-1473.

Snead, L.L., Nozawa, T., Katoh, Y., Byun, T.S., Kondo, S., Petti, D.A., 2007. Handbook of SiC properties for fuel performance modeling. J. Nucl. Mater. 371, 329-377.

Tekoglu, C., Pardoen, T., 2010. A micromechanics based damage model for composite materials. Int. J. Plast. 26, 549-569.

Tomičević, Z., Kodvanj, J., Hild, F., 2016. Characterization of the nonlinear behavior of nodular graphite cast iron via inverse identification analysis of uniaxial tests. Eur. J. Mech. - A/Solids 59, 140-154.

Tvergaard, V., 1981. Influence of voids on shear band instabilities under plane strain conditions. Int. J. Fract. 17, $389-407$.

Tvergaard, V., 1990. Analysis of tensile properties for a whisker-reinforced metal-matrix composite. Acta Metallurgica et Materialia 38, 185-194.

Tvergaard, V., Needleman, A., 1984. Analysis of the cup-cone fracture in a round tensile bar. Acta metall. 32, 157-169.

Vincent, P.G., Suquet, P., Monerie, Y., Moulinec, H., 2014. Effective flow surface of porous materials with two populations of voids under internal pressure: I. A GTN model. Int. J. Plast. 56, 45-73.

Voyiadjis, G.Z., Kattan, P.I., 1993. Local approach to damage in elasto-plastic metal matrix composites. Int. J. Damage Mech. 2, 92-114.

Williams, J.J., Flom, Z., Amell, A.A., Chawla, N., Xiao, X., De Carlo, F., 2010. Damage evolution in SiC particle reinforced Al alloy matrix composites by X-ray synchrotron tomography. Acta Mater. 58, 6194-6205. 